\title{
THE MEAN CONVERGENCE OF NON-HARMONIC SERIES
}

\section{HARRY POLLARD}

The theory of non-harmonic series deals with the completeness and expansion properties of sets of functions $\left\{e^{i \lambda_{n} x}\right\},-\infty<n<\infty$. Extending results of Paley and Wiener, Levinson has obtained a theory of these functions in the spaces $L^{p}, 1<p \leqq 2[5$, p. $113 ; 4$, p. 48$] .{ }^{1} \mathrm{His}$ principal result states that if

$$
\left|\lambda_{n}-n\right| \leqq \delta \quad(n=0, \pm 1, \cdots)
$$

with $\delta<(p-1) / 2 p, \lambda_{n}$ real, then any function $f(x) \in L^{p}$ has a Fourier expansion in the above functions equiconvergent with its ordinary Fourier series in any closed interval $(-\pi+\epsilon, \pi-\epsilon)$.

This, however, leaves open the question whether the expansion converges to $f(x)$ in the topology of $L^{p}(-\pi, \pi)$. This is known to be true for all $p>1$ if $\lambda_{n}=n[6$, p. 153]. For the non-harmonic case the only available results concern $p=2$ : by refining an earlier result of Paley and Wiener, Duffin and Eachus [2, p. 855] obtain mean convergence for $\delta<\pi^{-1} \log 2$. (This is less than the $\delta<1 / 4$ to be hoped for from Levinson's result.)

In this paper we shall generalize the Duffin-Eachus result to $L^{p}$, $p>1$. Our hypothesis is, however, more stringent than (1). We require that

$$
\left(\sum_{-\infty}^{\infty}\left|\lambda_{n}-n\right|^{a}\right)^{1 / a}=\delta<\pi^{-1} \log 2, \quad a=\frac{2 p}{|2-p|} .
$$

If $p \rightarrow 2$ this becomes sup $\left|\lambda_{n}-n\right|<\pi^{-1} \log 2$, which is the result we are extending.

It is interesting to observe that (2) remains the same if we replace $p$ by its conjugate $p^{\prime}$, where $1 / p+1 / p^{\prime}=1$. For then $|2 p /(p-2)|$ $=\left|2 p^{\prime} /\left(2-p^{\prime}\right)\right|$. We note also that the $\lambda_{n}$ need not be real.

TheOREM. Let $\left\{\lambda_{n}\right\}$ satisfy (2). Then any function $f(x) \in L^{p}, p>1$, admits a unique representation

$$
f(x)=\underset{N \rightarrow \infty}{\operatorname{lilm}} . \underset{-N}{N} \sum_{-N}^{N} c_{n} e^{i \lambda_{n} x}
$$

\section{Moreover}

Received by the editors January 31, 1944.

1 Numbers in brackets refer to the references listed at the end of the paper. 


$$
\begin{array}{ll}
\left(\sum_{-\infty}^{\infty}\left|c_{n}\right|^{p^{\prime}}\right)^{1 / p^{\prime}} \leqq\left(2-e^{\pi \delta}\right)-1\|f\|_{p} & (1<p \leqq 2), \\
e^{\pi \delta}\left(\sum_{-\infty}^{\infty}\left|c_{n}\right|^{p^{\prime}}\right)^{1 / p^{\prime}} \geqq\|f\|_{p} & (2 \leqq p<\infty),
\end{array}
$$

where $1 / p+1 / p^{\prime}=1$ and

$$
\|f\|_{p}=\left(\frac{1}{2 \pi} \int_{-\pi}^{\pi}|f(x)|^{p} d x\right)^{1 / p} .
$$

Levinson's methods are a combination of Fourier analysis and contour integration. We shall revert to the original real variable methods of Paley and Wiener, using the fact, observed by Boas, that their basic completeness theorem generalizes in a simple manner to general Banach spaces.

Boas' generalization, slightly amended, reads as follows [1, p. 469].

LEMMA 1. Let $\left\{x_{n}\right\},\left\{y_{n}\right\}$ be sequences in a Banach space $B$ such that for some $\lambda, 0<\lambda<1$, and all finite sequences $\left\{a_{n}\right\}$

$$
\left\|\sum a_{n}\left(x_{n}-y_{n}\right)\right\| \leqq \lambda\left\|\sum a_{n} x_{n}\right\| \text {. }
$$

Then if $\left\{x_{n}\right\}$ is a base, so is $\left\{y_{n}\right\}$. If $x \in B$ has the expansion $\sum_{1}^{\infty} c_{n} y_{n}$, then

$$
\frac{1}{1+\lambda}\|x\| \leqq\left\|\sum_{1}^{\infty} c_{n} x_{n}\right\| \leqq \frac{1}{1-\lambda}\|x\| .
$$

Lemma $2\left[3\right.$, p. 202]. If $f(x) \in L^{p}(-\pi, \pi)$ and $\left\{c_{n}\right\}$ are its Fourier coefficients, then

$$
\begin{array}{ll}
\left(\sum_{-\infty}^{\infty}\left|c_{n}\right|^{p^{\prime}}\right)^{1 / p^{\prime}} \leqq\|f\|_{p} & (1<p \leqq 2), \\
\left(\sum_{-\infty}^{\infty}\left|c_{n}\right|^{p^{\prime}}\right)^{1 / p^{\prime}} \geqq\|f\|_{p} & (2 \leqq p<\infty) .
\end{array}
$$

We remind the reader of our definition (5) of $\|f\|_{p}$.

LEMMA $3\left[3\right.$, p. 144]. If $f(x) \in L^{\text {s then }}$

$$
\|f\|_{r} \leqq\|f\|_{s} \quad(0<r \leqq s) .
$$

To prove our theorem we shall apply Lemma 1 with $x_{n}=e^{i n x}$, $y_{n}=e^{i \lambda_{n} x}, B=L^{p}$ (with modified norm (5)). (6) then becomes

$$
\left\|\sum a_{n}\left(e^{i n x}-e^{i \lambda_{n} x}\right)\right\|_{p} \leqq \lambda\left\|\sum a_{n} e^{i n x}\right\|_{p}
$$


We denote the right-hand side by $R$, the left-hand by $L$. Then we wish to show $L \leqq R$ for a value of $\lambda, 0<\lambda<1$.

Hence

$$
\begin{aligned}
L & =\left\|\sum a_{n} e^{i n x} \sum_{k=1}^{\infty} \frac{i^{k}\left(\lambda_{n}-n\right)^{k} x^{k}}{k !}\right\|_{p} \\
& =\left\|\sum_{k=1}^{\infty} \frac{i^{k} x^{k}}{k !} \sum a_{n}\left(\lambda_{n}-n\right)^{k} e^{i n x}\right\|_{p} .
\end{aligned}
$$

$$
L \leqq \sum_{k=1}^{\infty} \frac{\pi^{k}}{k !}\left\|\sum a_{n}\left(\lambda_{n}-n\right)^{k} e^{i n x}\right\|_{p} .
$$

Case (i). $1<p \leqq 2$. Then by Lemma 3

$$
\begin{aligned}
L & \leqq \sum_{k=1}^{\infty} \frac{\pi^{k}}{k !}\left\|\sum a_{n}\left(\lambda_{n}-n\right)^{k} e^{i n x}\right\|_{2} \\
& =\sum_{k=1}^{\infty} \frac{\pi^{k}}{k !}\left(\sum\left|a_{n}\left(\lambda_{n}-n\right)^{k}\right|^{2}\right)^{1 / 2} \\
& \leqq \sum_{k=1}^{\infty} \frac{\pi^{k}}{k !}\left(\sum\left|a_{n}\right|^{p^{\prime}}\right)^{1 / p^{\prime}}\left(\sum\left|\lambda_{n}-n\right|^{\left.k \cdot 2 p^{\prime} /\left(p^{\prime}-2\right)\right)^{\left(p^{\prime}-2\right) / 2 p^{\prime}}}\right. \\
& \leqq\left(\sum\left|a_{n}\right|^{p^{\prime}}\right)^{1 / p^{\prime}} \quad \sum_{k=1}^{\infty} \frac{\pi^{k}}{k !}\left(\sum_{-\infty}^{\infty}\left|\lambda_{n}-n\right|^{2 p^{\prime} /\left(p^{\prime}-2\right)}\right)^{k\left(p^{\prime}-2\right) / 2 p^{\prime}} .
\end{aligned}
$$

Also by Lemma 2

$$
R \geqq \lambda\left(\sum\left|a_{n}\right|^{p^{\prime}}\right)^{1 / p^{\prime}}
$$

Now write $\delta=\left(\sum_{-\infty}^{\infty}\left|\lambda_{n}-n\right|^{2 p^{\prime} /\left(p^{\prime}-2\right)}\right)^{\left(p^{\prime}-2\right) / 2 p^{\prime}}$. By (10) and (11), (8) is satisfied if

$$
e^{\pi \delta}-1<1, \quad \delta<\pi^{-1} \log 2 .
$$

But this is precisely our hypothesis (2).

Then the hypotheses of Lemma 1 are satisfied, (3) follows immediately, (4) is a consequence of (7) and the first part of Lemma 2.

Case (ii). $2 \leqq p<\infty$. We have by Lemma 2 and (9)

$$
\begin{aligned}
L & \leqq \sum_{k=1}^{\infty} \frac{\pi^{k}}{k !}\left(\sum\left|a_{n}\left(\lambda_{n}-n\right)^{k}\right| p^{\prime}\right)^{1 / p^{\prime}} \\
& \leqq \sum_{k=1}^{\infty} \frac{\pi^{k}}{k !}\left(\sum\left|a_{n}\right|^{2}\right)^{1 / 2}\left(\sum\left|\lambda_{n}-n\right|^{k \cdot 2 p^{\prime} /\left(2-p^{\prime}\right)}\right)^{\left(2-p^{\prime}\right) / 2 p^{\prime}} .
\end{aligned}
$$

Also by Lemma 3 


$$
R \geqq \lambda\left\|\sum a_{n} e^{i n x}\right\|_{2}=\lambda\left(\sum\left|a_{n}\right|^{2}\right)^{1 / 2} .
$$

From here on the argument is analogous to that of Case (i).

\section{REFERENCES}

1. Boas, R. P., Expansions of analytic functions, Trans. Amer. Math. Soc. vol. 48 (1940) pp. 467-487.

2. Duffin and Eachus, Some notes on an expansion theorem of Paley and Wiener, Bull. Amer. Math. Soc. vol. 48 (1942) pp. 850-855.

3. Hardy, Littlewood and P6lya, Inequalities, Cambridge, 1934.

4. Levinson, N., Gap and density theorems, New York, 1940.

5. Paley and Wiener, Fourier transforms in the complex domain, New York, 1934.

6. Zygmund, A., Trigonometrical series, Warsaw, 1935.

Kenyon College 